\title{
Itinéraires Itinéraires
}

Littérature, textes, cultures

\section{À la recherche d'une poétique ou comment lire une chanson populaire américaine}

\section{Frédéric Sylvanise}

\section{(2) OpenEdition}

1 Journals

Édition électronique

URL : http://journals.openedition.org/itineraires/2486

DOI : $10.4000 /$ itineraires.2486

ISSN : 2427-920X

Éditeur

Pléiade

\section{Référence électronique}

Frédéric Sylvanise, «À la recherche d'une poétique ou comment lire une chanson populaire américaine », Itinéraires [En ligne], 2014-2 | 2015, mis en ligne le 17 juillet 2015, consulté le 01 octobre 2020. URL : http://journals.openedition.org/itineraires/2486 ; DOI : https://doi.org/10.4000/itineraires. 2486

Ce document a été généré automatiquement le 1 octobre 2020.

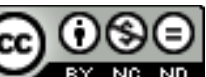

Itinéraires est mis à disposition selon les termes de la licence Creative Commons Attribution - Pas d'Utilisation Commerciale - Pas de Modification 4.0 International. 


\title{
À la recherche d'une poétique ou comment lire une chanson populaire américaine
}

\author{
Frédéric Sylvanise
}

\section{Introduction}

1 Le point de départ de nos travaux sur la poétique des chansons populaires américaines tient dans le constat suivant: il n'existe pas aujourd'hui de discipline universitaire totalement satisfaisante qui puisse appréhender une chanson de langue anglaise poétiquement. Encore faut-il s'entendre sur ce terme, que nous définirons plus loin. Par chanson nous entendons une forme hybride travaillée par trois éléments de nature fort différente qui sont : un texte, c'est-à-dire des « mots » comme dirait Nattiez, « ceux de la langue naturelle, mais aussi ceux que, plus particulièrement, nous rencontrons dans la littérature, la poésie, le récit, le mythe et le livret d'opéra, versifié ou non » (2010: 10); de la musique, soit les "sons organisés» (Ibid.: 9) des œuvres vocales des traditions populaires occidentales; et une ou des voix, auxquels il faudrait ajouter un quatrième élément subsidiaire dont l'importance varie selon les genres et les époques, à savoir la technologie (que l'on entendra essentiellement par le travail de studio, encore appelé production).

2 À l'heure actuelle, l'enseignement de la musique au sens large (et donc accessoirement, l'étude des chansons) prend deux formes bien distinctes à l'université, qui n'entretiennent pas ou peu de rapports entre elles : d'une part, la musicologie, c'est-àdire la science de la musique, qui s'intéresse à la théorie, à l'esthétique, et à l'histoire de la musique notamment. Elle a avant tout pour objet des caractéristiques techniques et évacue du même coup largement la problématique poétique des chansons. D'autre part, les cultural studies qui ont d'abord trouvé leur place dans les universités des pays anglophones et dans lesquelles l'accent est mis sur le contexte des chansons et sur les schémas culturels à l'œuvre dans les conditions de leur production. Il ressort 
généralement de ce type d'approche qu'il existe des déterminants extra-artistiques sur les créations. Mais, de ce fait, la notion de poétique est là aussi très souvent évacuée. Si le texte est étudié, en général comme un simple message, la voix, par exemple, n'y fait pas toujours l'objet d'une réflexion spécifique. Les deux domaines précités peuvent donc indéniablement être utiles au critique littéraire, mais ce dernier ne peut toutefois en tirer plus que de simples indications car ni l'un ni l'autre n'envisage les chansons comme un tout signifiant poétiquement, c'est-à-dire fonctionnant selon une logique propre.

3 Il est donc nécessaire de trouver des outils pour ne pas limiter l'étude de la poétique au seul texte, en fondant une démarche critique pragmatique qui permettrait une lecture des chansons, comme on parle de lecture des textes littéraires. Cela étant, le critique ne peut faire l'économie d'une étude poétique du texte de la chanson, en s'intéressant de près à la structure du texte, aux rimes, à la prosodie, à tout ce qui lui permet de s'acheminer vers une interprétation. Mais ce n'est qu'une étape, qui doit impérativement être pensée en fonction d'autres étapes. En France, le chercheur Stéphane Hirschi, dans un ouvrage intitulé Chanson, l'art de fixer l'air du temps: de Béranger à Mano Solo, a développé une théorie proche de celle que nous préconisons, appelée "cantologie». En considérant la chanson comme un "tout organique " (Hirschi 2008 : 25), l'auteur voit juste. Il lui apparaît impossible de séparer les éléments constitutifs d'une chanson lorsqu'on en fait l'étude: «il s'agit bien de considérer l'œuvre chanson globalement, comme une rencontre entre un texte, une musique et une interprétation" (Ibid.). Nous verrons par la suite que si le texte et la musique sont effectivement des éléments essentiels à prendre en compte, la notion d'interprétation nous paraît un peu moins opérante que celle de voix, car si l'interprétation englobe tout le corps, elle le fait essentiellement lors de concerts. Or, la plupart des chansons que nous nous proposons d'étudier ont été enregistrées en studio, où la notion d'interprétation (et de " performance ${ }^{1}$ ", employée également par Hirschi) est presque secondaire. Mais il s'agit ici d'une différence de degré plus que de structure. Il va de soi que dans un concert, le corps s'exprime de manière autrement plus intense que dans un studio où les erreurs peuvent être corrigées et les prises multipliées qui plus est... En revanche, lorsqu'il procède à une différenciation entre chanson à la française et song à l'anglo-saxonne, Hirschi avance des arguments discutables :

$\mathrm{Au}$ sein de l'intrication texte-musique-interprétation sur fond de temps compté, la majorité des morceaux de la production anglophone relève en effet d'une autre esthétique dominante. On peut à leur sujet parler de dilution: les paroles interprétées - quelles que soient leurs qualités intrinsèques - s'y fondent dans la musique [...]. (Hirschi $2008: 26$ )

Outre que cette généralisation nous semble excessive, les arguments avancés par l'auteur pour l'étayer sont déroutants. En effet, pour illustrer son propos, Hirschi prend pour exemple la version anglaise (donc traduite) d'un opéra-rock de Michel Berger (Starmania). Non seulement la version anglaise d'un texte français ne nous semble pas devoir être représentative de la tradition anglophone, mais le raisonnement avancé par Hirschi pour distinguer les deux traditions est largement induit par les aléas du texte d'arrivée forcément soumis à caution (Hirschi $2008: 26-28$ ). Même s'il reconnait que des contre-exemples existent à sa théorie ${ }^{2}$ sur ce qu'il appelle song, il peine à convaincre faute d'expliquer clairement ce qui fait la spécificité de la chanson anglophone par rapport à la chanson francophone. Si la cantologie peut donc s'appliquer à cette dernière, elle est dans une impasse pour étudier la première. 
Pour préciser ce que nous entendons par poétique, nous partirons de la définition qu'en donne Henri Meschonnic dans Pour la poétique I, soit l'étude de la littérarité comprise comme "spécificité de l'œuvre comme texte ; ce qui le définit comme espace littéraire orienté, c'est-à-dire une configuration d'éléments réglée par les lois d'un système » (Meschonnic 1970 : 174). Si l'on admet qu'une chanson forme également un système, qui certes n'est pas fixe car il peut y en avoir plusieurs interprétations, alors la notion de texte chez Meschonnic pourra être remplacée par celle de chanson dans notre perspective. En effet, en studio, l'œuvre est finie, achevée, tout comme le texte littéraire. On peut alors considérer notre objet d'étude lui aussi comme une sorte de texte à trois voire à quatre entrées, qu'il faut apprendre à lire en les mettant en relation les unes avec les autres.

6 À cette notion de poétique, on peut adjoindre celle de «forme-sens » en suivant là encore Meschonnic, qui la définit comme « forme du langage dans un texte (des petites aux grandes unités) en tant que produit de l'homogénéité du dire et du vivre. Un texte, dans son signifiant est l'inconscient du langage. Il fait ceci, qu'il dure, et on ne peut pas en épuiser le pourquoi. Sa connaissance est infinie" (Meschonnic 1970: 176). Dans notre perspective, nous pourrions considérer que dans une chanson, le timbre des instruments, la mélodie, certains types de rythme, la qualité d'une voix (que l'on appellera plus tard son grain) et éventuellement les modifications que la technologie peut leur apporter, constituent l'inconscient du langage autant que le texte écrit (ce que l'on nomme les lyrics en anglais) de cette chanson, c'est-à-dire que tous ces éléments participent pleinement de sa signification, en jouant les uns avec les autres et parfois les uns contre les autres (surtout dans le cas de chansons où l'ironie joue à plein).

7 Dans un premier temps, nous verrons ce qui rapproche et ce qui sépare le langage musical du langage littéraire. Nous tenterons de déterminer ce que la critique littéraire peut faire du langage musical lorsqu'elle veut l'analyser en rapport avec les autres éléments d'une chanson. Puis, nous tâcherons d'aborder la question encore plus périlleuse de l'étude de la voix, en utilisant notamment les intuitions de Roland Barthes sur ce qu'il appelle sa « signifiance ». Enfin, la technologie fera l'objet de notre dernier développement. Nous nous intéresserons non seulement à la manière dont elle peut transformer une chanson, mais verrons qu'elle est aussi parfois partie intégrante de l'essence même de certains genres musicaux. À chaque fois, nous tâcherons de fournir quelques brefs exemples d'analyses possibles de chansons afin d'éclairer nos réflexions théoriques, en puisant dans un corpus américain essentiellement contemporain, soit notre domaine de recherche depuis une dizaine d'années maintenant.

\section{Texte et musique}

$8 \mathrm{Si}$ « lire » une chanson ne va pas de soi, c'est d'abord parce que le langage littéraire et le langage musical sont de nature différente, comme on le verra. À première vue pourtant, comme le souligne André Spire :

[...] il y aurait folie à nier les analogies entre la musique et « sa sœur articulée » la poésie.

Non seulement elles ont un domaine commun, celui des sons et des rythmes, mais elles ont mêmes intentions, tendent aux mêmes buts. Toutes deux sont la manifestation d'un même désir plus ou moins avoué ou secret: au moyen de combinaisons rythmico-sonores, s'emparer du monde des choses ou du monde des 
êtres ravis et dominés par la délectation d'un plaisir d'origine physique éprouvé sur soi-même, et censé communiqué ou réellement communiqué.

De plus, toutes deux sont issues du même complexe dynamique primitif : geste, cri plus ou moins modulé, parole plus ou moins articulée, par lequel l'organisme humain répondait aux excitations d'origine extérieure ou intérieure, elles furent plus tard et longtemps confondues dans une activité indivise: musique-poésiedanse qui apparaît d'abord dans des cérémonies magiques. Ce groupe peu à peu se dissocia en combinaisons binaires : musique-parole (le chant), musique-geste (la danse, la pantomime), paroles-gestes (le théâtre), d'où se détacha, par élimination progressive de tous les autres éléments, une musique sans contenu apparent de paroles ou de gestes, la musique prétendue pure, et qu'il me paraît préférable, avec Lionel Landry, d'appeler musique autonome. (Spire 1948 : 7)

Cette communauté d'origine entre les deux langages se double d'une communauté terminologique, car la critique littéraire et la critique musicale utilisent parfois les mêmes mots, ce qui occasionne des confusions et des erreurs : des notions telles que le rythme, le ton, la cadence sont utilisées par elles sans toutefois recouvrir les mêmes phénomènes (Spire 1948: 6). En outre, certains écrivains n'hésitent pas à emprunter à la musique le titre de leurs œuvres: Gide avec La Symphonie pastorale (1919) ou, plus près de nous, Toni Morrison avec Jazz (1993) par exemple, tandis que l'imaginaire littéraire de la musique est évident, ne serait-ce que dans les multiples mises en musique d'œuvres littéraires (on pense surtout à l'opéra et aux poèmes symphoniques). Les ponts entre les deux champs sont donc nombreux et peuvent se traverser dans les deux sens, mais il faut approfondir l'analyse de leur essence pour mieux comprendre leurs différences. Aude Locatelli peut nous y aider, qui conclut ainsi une étude consacrée au rapport entre littérature et musique au siècle dernier :

La confrontation de la musique et la littérature au $\mathrm{xx}^{\mathrm{e}}$ siècle fait en somme ressortir non seulement les similitudes mais aussi les spécificités de ces deux systèmes sémiotiques, non réductibles au seul lien de complémentarité. Elle implique nécessairement une interrogation sur leurs caractéristiques respectives et ne peut s'effectuer sans que soient prises en compte la problématique des rapports entre la musique et le langage ainsi que l'ambivalence du champ musico-littéraire qui tient d'une part à la musicalité intrinsèque du mot et d'autre part à l'expressivité de la musique, apte à signifier, même si elle peut être qualifiée d'a-sémantique. (Locatelli $2001: 120)$

10 La différence de nature entre les deux domaines est donc patente, notamment lorsqu'on envisage leur rapport à la signification (Lévi-Strauss 1971:580; Barthes 1982 : 236). Comme l'explique précisément Françoise Escal :

[...] l'expressivité musicale libère un sens mais ne fournit pas de significations à proprement parler: son immanence à la forme empêche de définir les relations entre signifiant et signifié de la même façon que dans le langage verbal. Le sens musical, quand il est traduit par des mots, est abusivement converti en significations trop précises et littérales. Il n'y a pas d'équivalent verbal de la forme musicale. La musique n'a pas de fonction référentielle en ce que, si une référence existe, elle ne s'impose pas à tous les sujets d'une même culture ou d'une même société, à toutes les époques (sauf cas rares). [...] Lorsqu'il y a une cohérence des interprétations sémantiques d'un même extrait musical, cette cohérence n'exprime qu'une signification dominante, non exclusive d'autres significations pouvant paraitre parfois contradictoires. (Escal $1990: 104$ )

11 De plus, comme l'affirme judicieusement Éric Dufour, «la musique ne possède nul pouvoir sémantique véritable puisqu'elle ne reçoit de sens que par l'intermédiaire du langage verbal» (Dufour 2005: 58, note 2). Par «sémantique», Dufour entend 
référentiel. Autrement dit, sans minimiser le pouvoir évocateur de la musique, l'orage qu'on entend dans la sixième symphonie de Beethoven est un orage avant tout parce que Beethoven nous a donné un programme. De même dans le "Livery Stable Blues » de l'Original Dixieland Jass Band (OJDB 1917 : piste $n^{\circ} 1$ ) ou le « Machine Gun ${ }^{3}$ » de Jimi Hendrix (1970: piste $n^{\circ}$ 2), on reconnaît les cris des animaux de la ferme ou le bruit des mitraillettes de la guerre du Vietnam parce que le titre des morceaux nous y invite. On pourrait d'ailleurs parfaitement renverser cette affirmation de Locatelli :

La revendication d'une écriture musicale, qui fait le plus souvent partie du paratexte, ne permet évidemment pas à elle seule d'affirmer la possibilité d'une contribution de la musique à la création romanesque. Cependant elle témoigne indéniablement d'une demande de la part de la littérature envers les modèles formels offerts par la musique. (Locatelli $2001: 85$ ) amené à s'engager sur la voie de la métaphore ou de la comparaison pour faire jouer le discours musical avec les autres éléments constitutifs de la chanson. Bien sûr, on entrevoit les limites d'un tel exercice qui est descriptif avant que d'être à proprement parler analytique. Escal l'a bien senti, qui y voit l'un des travers de la critique musicale :

Cette attitude critique qui consiste à faire part au public des images que suscite une œuvre musicale peut prêter le flanc... à la critique. Pour certains, cela revient à traduire la musique sous la catégorie verbale la plus pauvre ou la plus arbitraire : l'adjectif. [...] Compte tenu de la nature non significative de la musique (elle a du sens mais pas ou peu de signes), la prédication prend ainsi la forme la plus gratuite : l'épithète. Pour Barthes, ce recours à l'épithète prédicative peut s'interpréter comme le rempart dont l'imaginaire du critique et/ou du sujet social se protège de la perte dont il est menacé par cet art d'aucun usage pratique communicatif; la musique, champ de signifiance et non système de signes comptables, échangeables, fait du musicien un fou, alors que l'écrivain est condamné à la signification. (Escal $1990: 103-104)$

Selon Locatelli (2001: 87), un « danger de la "mauvaise" métaphore » menacerait même le critique littéraire qui s'aventure à évoquer ce que la musique lui fait ressentir. Karol Beffa est plus radical encore :

Après tout, pourquoi parler de musique ? Ne se suffit-elle pas à elle-même ? En quoi un discours sur la musique permettrait-il de mieux la comprendre ou de mieux l'interpréter? En quoi permettrait-il de mieux l'entendre et d'en jouir davantage? Le problème est classique et a ses parallèles dans d'autres domaines. Mais la difficulté est ici redoublée par le fait que la musique est un art peu mimétique et que la question du sens s'y pose en des termes bien particuliers. Contrairement à la littérature, qui use du même medium verbal que son objet, le discours sur la musique - qu'il soit technique ou métaphorique -, empruntant un medium qui ne se situe pas sur le même plan que la musique elle-même, n'est pas assuré de pouvoir saisir quelque chose de son objet, ressenti comme irréductible par essence et, semble-t-il, voué à lui échapper. D'une certaine façon, la musique met en demeure 
le langage de se plier à sa particularité - pour être en quelque sorte à sa hauteur.

(Beffa 2013 : 21-22) Oui, s'il n'a affaire qu'à la musique seule, mais sans doute moins lorsqu'il étudie une chanson, car il ne peut faire abstraction de ses autres éléments constitutifs. Il lui faut décrire, certes, mais aussi réfléchir en termes sémiotiques à la fonction de la musique par rapport aux voix et au texte. C'est donc moins la musique en soi qui l'intéresse que la musique dans un dialogue avec les voix et le texte. La musique seule n'a peut-être pas de signifié, mais dans le système de la chanson, elle peut tout à fait être lue comme un signe qui joue avec d'autres signes. Et somme toute, la métaphore, comme le souligne Tagg (2012 : 79-80), est le moyen le plus commode de décrire les émotions suscitées par la musique chez l'auditeur et le point de départ à des analyses plus poussées.

Parmi les multiples exemples susceptibles d'illustrer la richesse du dialogue entre texte et musique pris comme signes, la chanson «Rednecks ${ }^{4}$ » de Randy Newman (1974 : piste $n^{\circ} 1$ ) est particulièrement intéressante. La première partie (jusqu'à $1^{\prime} 35$ ), qui inclut trois couplets et le refrain, met en évidence le ton à la fois ironique (car le chanteur parle à la première personne et endosse donc le rôle-titre) et sarcastique de Newman. La persona $a^{5}$ est en effet un homme du sud qui joue le rôle de porte-parole de tous les rustres (le terme « rednecks » désignant à l'origine le cou des travailleurs agricoles du sud des États-Unis rougi par le soleil) :

Last night I saw Lester Maddox on a TV show

With some smart ass New York Jew

And the Jew laughed at Lester Maddox

And the audience laughed at Lester Maddox too

Well he may be a fool but he's our fool

If they think they're better than him they're wrong

So I went to the park and I took some paper along

And that's where I made this song

We talk real funny down here

We drink too much and we laugh too loud

We're too dumb to make it in no Northern town

And we're keepin' the niggers down

We got no-necked oilmen from Texas

And good ol' boys from Tennessee

And colleges men from LSU

Went in dumb. Come out dumb too

Hustlin' 'round Atlanta in their alligator shoes

Getting' drunk every weekend at the barbecues

And they're keepin' the niggers down

CHORUS

We're rednecks, rednecks

And we don't know our ass from a hole in the ground

We're rednecks, we're rednecks

And we're keeping the niggers down

Traduction $^{6}$

Hier soir je regardais Lester Maddox dans une émission de télé

Avec un Juif new-yorkais qui se croit malin

Et le Juif s'est moqué de Lester Maddox

Et la salle aussi s'est moquée de Lester Maddox

Bon, c'est peut-être un idiot, mais c'est notre idiot à nous

S'ils pensent qu'ils valent mieux que lui ils se trompent 


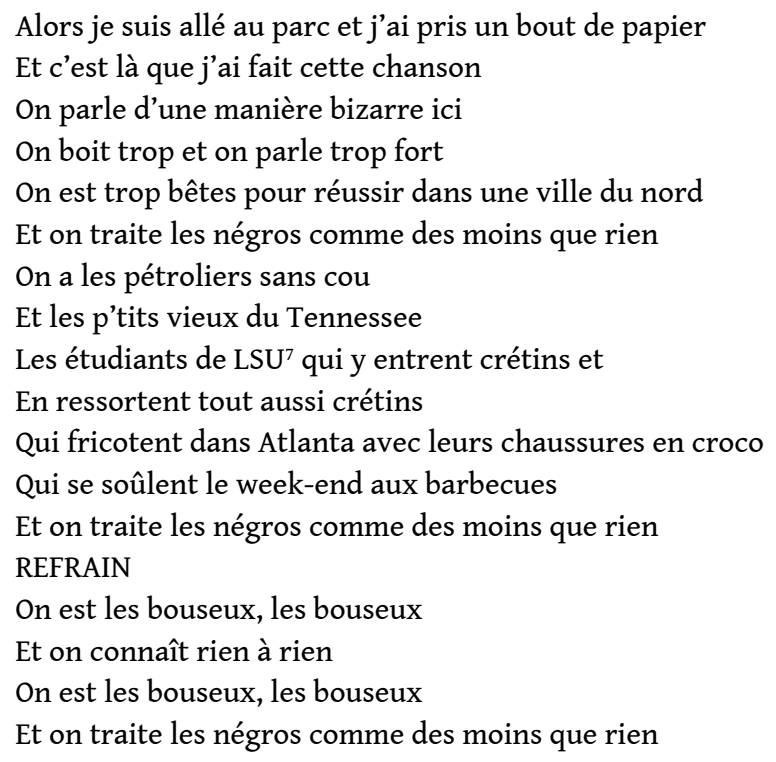

Le portrait que fait Newman de sa persona est tout sauf élogieux: bêtement conservateur, raciste, antisémite, inculte, il cumule les tares. La musique fonctionne comme un écho caricatural à ce texte qui ne l'est pas moins. En effet, c'est un jazz néoorléanais décanté qui accompagne les trois premières strophes, la clarinette jouant un rôle prépondérant à partir de la deuxième strophe, évoquant un Sidney Bechet de carte postale. Quant au refrain, il est chanté faux par tous les musiciens. Musique et texte fonctionnent ici en miroir et produisent un effet comique très efficace. En opérant une sorte de synthèse musicale facilement identifiable pour l'auditeur moyen, on pourrait dire une vignette musicale, Newman fait dire à sa persona qu'il parle depuis le sud profond. En le faisant chanter faux, il souligne son caractère grossier et inculte, déjà très sensible dans le texte. D'une certaine manière, la musique de Newman, qui relève un peu du cliché, vient souligner le discours politique mal dégrossi de la persona qui relève lui aussi du cliché sur le sud (la seconde moitié de la chanson s'attaque d'ailleurs à l'arrogance des gens du nord avec autant de verve et une ironie au carré, faisant du racisme une sorte d'universel américain). La puissance de «Rednecks » n'est donc pas liée à son seul texte, dont la charge sarcastique est en soi d'une grande violence, mais à la manière de mettre en scène ce texte avec une musique conçue comme un pot-pourri de la «norme » musicale du sud et avec une voix nasillarde et traînante qui évoque l'accent du sud des États-Unis. C'est justement à la voix et à ses pouvoirs que nous allons nous intéresser à présent, afin de mieux comprendre son importance au sein de la forme-sens qu'est la chanson.

\section{Étudier la voix}

19 Si la question de la technique est centrale dans l'étude de la musique, elle semble moins décisive lorsqu'on examine une voix. Il nous apparaît en effet difficile de s'arrêter à un discours purement technique sur la voix, qui est problématisée par bien des disciplines, comme le notait Roland Barthes dans un entretien accordé à Hector Bianciotti :

Beaucoup de sciences, beaucoup de disciplines sont intéressées par la voix: la physiologie, l'esthétique bien sûr, mais aussi la psychanalyse, la sémiologie (comment une voix peut-elle signifier ?) et même la sociologie : il y a un lien entre les classes sociales et les types de voix; l'idéologie est présente dans la voix, et 
même la mode, qui porte souvent sur des objets réputés naturels. [...] ce qui m'intéresse le plus dans la voix, c'est que cet objet très culturel est, d'une certaine façon, un objet absent (beaucoup plus que le corps qui, lui est représenté de mille manières par la culture de masse) : nous écoutons rarement une voix "en soi ", nous écoutons ce qu'elle dit; la voix a le statut même du langage, qui est un objet que l'on croit ne pouvoir saisir qu'à travers ce qu'il véhicule; mais de même qu'aujourd'hui, grâce à la notion de "texte ", nous apprenons à lire la matière même du langage, de même il nous faudra apprendre à écouter le texte de la voix, sa signifiance, tout ce qui, en elle, déborde la signification. (Barthes 1973 : 488)

Comme face à la musique, le non-spécialiste est bien en mal d'aborder une voix autrement que métaphoriquement : « Le grain d'une voix n'est pas indicible (rien n'est indicible), mais je pense qu'on ne peut le définir scientifiquement, car il implique un certain rapport érotique entre la voix et celui qui l'écoute. On peut donc décrire le grain d'une voix, mais seulement à travers des métaphores " (Barthes $1973: 489$ ). Le cas du scat, cette technique vocale jazzistique qui consiste à égrener des onomatopées sur tous les tons possibles, est un peu l'exemple de l'expressivité de la voix poussée à son comble, une sorte de chant désintéressé, à l'état pur. Comme l'explique le critique canadien John Sobol, prolongeant ainsi la réflexion de Barthes en l'appliquant spécifiquement au jazz :

Because jazz vocalists in the 30 s were generally restricted to singing songs whose lyrics had little intrinsic poetic value to the black community, they found other ways to make their public voices as meaningful as possible. In scat singing, black oralists found a means of escape, a playful arena where their improvisatory urges could be given free rein and they could explode the restrictive limitations of banal lyrics'. (Sobol $2002: 36$, nous soulignons)

21 Mais, ici encore, c'est davantage en rapport avec la musique et le texte que l'on doit envisager la voix, et non pas en l'isolant. En effet, la voix est très exactement ce qui lie texte et musique car elle prononce des paroles (sauf, justement, dans le cas du scat précédemment évoqué) tout en se situant sur une échelle de notes. Surtout, elle atténue les différences entre langage musical et langage poétique. En effet, Spire a montré avec des chiffres que les possibilités de la poésie étaient moindres que celles de la musique du point de vue des durées, de l'intensité et de l'intonation (Spire 1948: 184-185). Or, dans une chanson, la voix porte le texte, mais elle le fait avec le support de la musique. Elle ne dit pas le texte (sauf dans le cas du spoken word, mais c'est une exception), mais elle le chante, c'est-à-dire qu'elle fait se rejoindre texte et musique ${ }^{9}$. Elle est donc à la fois l'incarnation de la musique et du texte puisqu'elle en opère la fusion.

L'expressivité de la voix trouve une illustration spectaculaire avec l'artiste britannique installé aux États-Unis Antony Hegarty et son groupe the Johnsons. Dans une chanson intitulée « For Today I'm a Boy ${ }^{10}$ » (Antony and the Johnsons, 2005 : piste $\mathrm{n}^{0}$ 3), le texte fait clairement allusion à l'ambiguïté sexuelle ${ }^{11}$ de la jeune persona, comme dans la première strophe et le refrain :

One day I'll grow up, I'll be a beautiful woman.

One day I'll grow up, I'll be a beautiful girl.

One day I'll grow up, I'll be a beautiful woman.

One day I'll grow up, I'll be a beautiful girl.

But for today I am a child, for today I am a boy.

For today I am a child, for today I am a boy.

For today I am a child, for today I am a boy. 


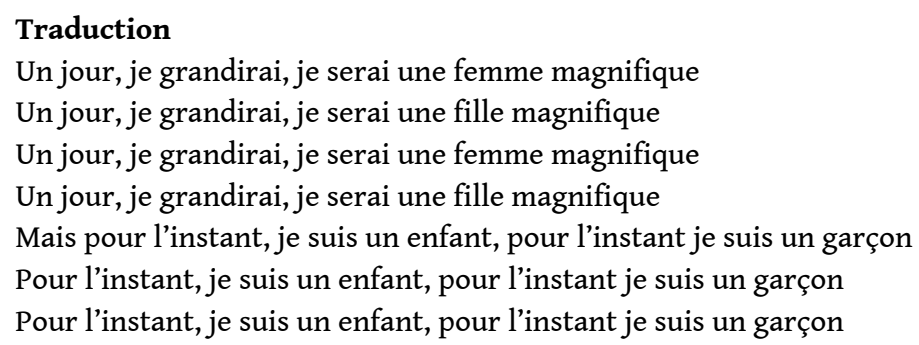
sexuelle fondamentale de la voix du chanteur. Au vacillement de la persona entre pôle masculin (le présent) et pôle féminin (l'avenir désiré), énoncé on ne peut plus explicitement par les paroles, fait écho l'androgynie d'une voix insaisissable qui évoque les chanteurs de gospel et Nina Simone. Pour reprendre l'idée de Barthes, le «texte de la voix » est ici au moins aussi signifiant que le texte chanté par la voix, comme si cette dernière était déjà, en soi, le signe absolu de l'ambiguïté sexuelle. La limite de la chanson tient donc dans le caractère presque redondant du texte par rapport à la pure expressivité de la voix. Il n'empêche que l'émotion qu'elle suscite est sincère car le grain de la voix de l'artiste est d'abord la traduction déchirante d'une forme de dilemme intime (jusqu'à quel point se dire garçon plutôt que fille ?). La voix d'Antony dans «For Today I'm a Boy » est doublée par un processus d'écho qui amplifie encore l'impression de dualité vocale du chanteur, ce qui nous amène naturellement à nous interroger sur le rôle de la technologie dans la chanson.

\section{La technologie ou la « valeur ajoutée » du studio}

Nul ne peut nier aujourd'hui l'importance de la technologie dans la création d'une chanson. Ce qu'on appelle la production n'a cessé de prendre de l'envergure au cours $\mathrm{du} \mathrm{xx}^{\mathrm{e}}$ siècle. Simple moyen de retransmission au départ, elle est devenue peu à peu déterminante, surtout à partir des années 1960. Certains genres musicaux, qui ont produit de grandes chansons, ont peu utilisé la technologie, parce qu'ils sont nés à des époques où elle était peu développée ou simplement parce qu'ils n'en avaient pas particulièrement besoin : on pense au blues, au folk, à la country. En revanche, le rock, le rap et aujourd'hui les musiques électroniques en ont fait une dimension essentielle $\mathrm{du}$ processus créatif, quitte à faire parfois passer la musique pour une affaire de machines plus que de musiciens. Aussi faut-il s'entendre sur ce qu'on appelle technologie : non pas le son lui-même, mais la mise en son.

Dans un article publié en 1992, le journaliste Gilles Tordjman a mesuré tout ce que la technologie avait apporté au rock (à prendre au sens large, et non pas au sens de rock'n'roll, genre musical qui naît au milieu des années 1950 à la suite du rythm'n'blues) :

[...] le duo Les Paul/Mary Ford est une des choses les plus atypiques qu'il nous ait été donné d'entendre : parce qu'il soumet l'existence de la musique aux conditions technologiques de sa production, il est absolument moderne. [...] Le rock n'est pas un genre, encore moins un langage : c'est avant tout une certaine manière d'utiliser l'outil musical. C'est un geste instrumental avant d'être une musique. Parce que, des Beatles à Music for a New Society, les disques de rock les plus bouleversants sont d'abord des expériences de studio, l'enregistrement multipistes échappe au domaine de la technique pour s'imposer comme l'élément fondamental d'une éthique musicale: ce qui explique l'importance de Good Vibrations sur un plan 
historique, c'est la rencontre parfaite d'un projet et d'une technique dont les limites sont sans cesse repoussées. Le studio moderne n'est peut-être qu'un outil. Mais c'est un outil qui, en faisant advenir l'inouï, bouscule totalement l'économie créatrice : le pire des instrumentistes peut s'y révéler le plus grandiose des artistes. (Tordjman $1992: 28)$

Lire une chanson suppose donc très souvent de voir comment la technologie influe sur la musique et la voix, c'est-à-dire comment, à son tour, elle est signifiante. Si elle est plus qu'un gadget, c'est que son pouvoir est immense, au risque du brouillage. Tel album est parfois jugé raté au seul motif que sa production est mauvaise (on pense notamment au Death of a Ladies' Man de Leonard Cohen, paru en 1977). En permettant des répétitions, des échantillonnages, des effets sonores très particuliers, elle révolutionne notre manière d'écouter et donc de lire une chanson ${ }^{12}$. On ne peut concevoir par exemple de rock psychédélique sans pédale wah-wah ni autre fuzz. Un artiste américain comme Don Van Vliet, autrement connu sous le nom de Captain Beefheart, a tenté dès son premier album intitulé Safe as Milk d'utiliser toutes les ressources possibles du studio, notamment avec les guitares électriques. Une chanson comme «Electricity ${ }^{13}$ » (Captain Beefheart 1967: piste $n^{\circ} 6$ ) est passionnante à lire parce qu'elle permet de faire le lien entre musique, voix et texte non seulement sur un plan thématique mais structurel. En voici les trois premières strophes, très influencées par le surréalisme :

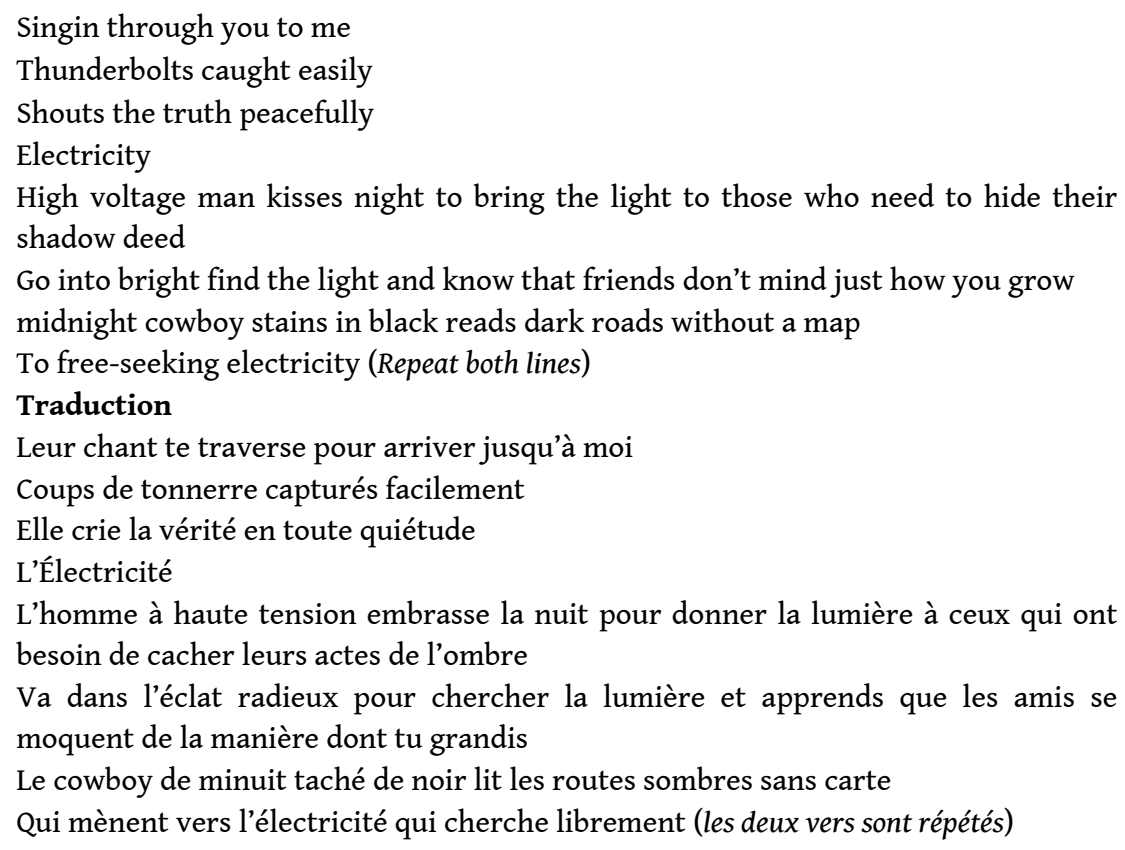

Ce qui frappe à l'écoute du morceau, c'est l'aspect tantôt caverneux, tantôt strident de la voix de Captain Beefheart. Elle devient un instrument au même titre que les autres et notamment les guitares électriques auxquelles les nombreux effets de distorsion opérés par la technologie donnent un aspect particulièrement agressif, encore accentué par le rythme syncopé qui suit le premier couplet. Cette voix monstrueuse et effrayante évoque bien entendu l'électricité du titre et le « high voltage » du premier couplet mais elle matérialise aussi de manière très concrète, par les multiples torsions qu'elle s'impose, les onomatopées du troisième couplet, si bien qu'on ne sait plus si la voix chante un texte pré-écrit ou si le texte tente tant bien que mal de rendre compte de la performance vocale du chanteur. De simple relais d'un texte, la voix devient ici une 
sorte de conducteur électrique qui retransmet le texte comme on dirait d'une radio qu'elle retransmet un reportage.

Le critique qui entreprend d'inventer une poétique des chansons se doit donc de chercher dans le «geste instrumental» ce qui modifie la musique en profondeur, ou simplement en surface, quand il modifie quelque chose. Une technique comme l'échantillonnage (autrement appelé sampling), apparue à la toute fin des années 1960 avec l'album Canaxis du Hongrois Holger Czukay, a peu à peu révolutionné la musique contemporaine, et notamment le genre rap, comme l'ont par exemple montré en France Christian Béthune puis Maxence Déon. L'échantillonnage est bien à l'origine d'une nouvelle forme de créativité fondée entre autres sur la répétition. Si elle n'est pas d'essence musicale au départ, cette technique transforme la musique en profondeur.

\section{Conclusion}

Une poétique de la chanson implique inévitablement d'inventer des analogies et des métaphores pour en saisir le fonctionnement et dépasser le seul repérage technique, mais pour bien lire une chanson, il faut d'abord bien l'écouter, c'est-à-dire se déprendre d'une tentation «logocentrique ». La musique et la voix ne sont pas là pour mettre un texte en son(s), pas plus qu'il n'y a d'ordre dans la manière de concevoir une chanson. Une mélodie peut parfaitement précéder l'écriture du texte et l'auteur n'est pas forcément le compositeur qui peut ne même pas connaître l'interprète de ladite chanson. En bref, lire une chanson suppose de ne pas hiérarchiser ses composantes et de ne pas en faire l'analyse en les isolant les unes des autres. On l'aura compris, le verbe lire dans l'expression "lire une chanson» signifie en fait lier. La chanson, en tant qu'hybride, suppose un dialogue permanent entre trois ou quatre éléments qui ne sont pas de même nature et une chanson réussie l'est justement parce qu'elle parvient à un équilibre qui peut être fragile. Enfin, le récepteur est également un créateur et doit faire non seulement appel à son oreille, mais aussi à sa mémoire et à sa culture car une chanson renvoie à d'autres chansons et à d'autres histoires, comme en littérature. C'est à lui de faire le départ entre ce qui était déjà là et ce qui relève de l'inouï.

\section{BIBLIOGRAPHIE}

Auslander, Philip, 1999, Liveness: Performance in A Mediatized Culture, Abingdon, Routledge.

-, 2009, « Physical persona: the physical performance of popular music », dans Derek B. Scott, The Ashgate Research Companion to Popular Musicology, Farnham, Ashgate, p. 303-315.

Barthes, Roland, 1982, L'Obvie et l'Obtus, Paris, Seuil.

-, [1973] 2002, « Les fantômes de l'Opéra », CEuvres complètes, t. IV, Paris, Seuil, p. 488-496.

_, [1972] 2002, « Le grain de la voix », Euvres complètes, t. IV, Paris, Seuil, p. 148-156.

Beffa, Karol, 2013, Comment parler de musique ?, Paris, Arthème Fayard et Collège de France. 
Béthune, Christian, 1999, Le Rap, une esthétique hors-la-loi, Paris, Autrement.

Chabot-Canet, Céline, 2008, Léo Ferré : une voix et un phrasé emblématiques, Paris, L’Harmattan.

Danielsen, Anne, 2010, Musical Rhythm in the Age of Digital Reproduction, Farnham, Ashgate.

Deniot, Joëlle et Dutheil, Catherine, 2000, «Chansons populaires, nuances féminines », dans Joëlle Deniot, Catherine Dutheil et François-Xavier Vrait (dir.), Dire la voix : approche transversale des phénomènes vocaux, Paris, L'Harmattan, p. 213-234.

Déon, Maxence, 2011, «L'échantillonnage comme choix esthétique : l'exemple du rap », Volume! La revue des musiques populaires, vol. 8, n 1, p. 277-301, [En ligne], http://volume.revues.org/1307.

Dufour, Éric, 2005, Qu'est-ce que la musique ?, Paris, Vrin, coll. « Chemins philosophiques ».

Escal, Françoise, 1990, Contrepoints : musique et littérature, Paris, Klincksieck.

Hirschi, Stéphane, 2008, Chanson, l'art de fixer l'air du temps : de Béranger à Mano Solo, Paris, Les Belles Lettres.

Lacasse, Serge, 1999, «La mise en scène de la voix en musique rock enregistrée : résultats préliminaires ", Les cahiers de la Société québécoise de recherche en musique, n 2 (1), p. 9-18.

-, 2005, « La musique populaire comme discours phonographique : fondements d'une approche analytique ", Musicologies, $\mathrm{n}^{\circ} 2$, p. 23-39.

Lacasse, Serge et Roy, Patrick, 2005, Groove : enquêtes sur les phénomènes musicaux contemporains, Québec, Presses de l'université Laval.

Lebrun, Barbara (dir.), 2012, Chanson et performance : mise en scène du corps dans la chanson française et francophone, Paris, L'Harmattan.

Lévi-Strauss, Claude, 1971, Mythologiques, t. IV, Paris, Plon.

Locatelli, Aude, 2001, Littérature et musique au Xx siècle, Paris, PUF, coll. « Que sais-je ?».

Meisel, Perry, 2009, The Myth Of Popular Culture, New York, Wiley-Blackwell.

Meschonnic, Henri, Pour la poétique I, Paris, Gallimard.

Middleton, Richard, 1990, Studying Popular Music, Philadelphie, Open University Press.

-, 2007, « Mum's the Word », dans Freya Jarman-Ivens (dir.), Oh Boy!: Masculinities and Popular Music, Abingdon, Routledge, p. 103-124.

Moore, Allan F., 2012, Song Means: Analysing and Interpreting Reorded Popular Song, Farnham, Ashgate.

Nattiez, Jean-Jacques, 2010, La musique, les images et les mots : du bon et du moins bon usage de la métaphore dans les esthétiques comparées, Québec, Fides.

Negus, Keith, 1996, Popular Music in Theory: An Introduction, Cambridge, Blackwell.

Schmidt-Horning, Susan, 2013, Chasing Sound: Technology, Culture, and the Art of Studio Recording from Edison to the LP, Baltimore, Johns Hopkins University.

Sobol, John, 2002, Digitopia Blues: Race, Technology, and the American Voice, Banf, Banff Centre Press. Spire, André, [1948] 1986, Plaisir poétique, plaisir musculaire, essai sur l'évolution des techniques poétiques, Paris, José Corti.

Tagg, Philip, 2012, Music's Meanings: A Modern Musiclogy for Non-Musos, New York, The Mass Media Music's Scholar Press. 
Tordjman, Gilles, 1992, « Les Paul vient d'inventer la pop-anglo-saxonne », Les Inrockuptibles, n 40 , p. 28.

Zagorski-Thomas, Simon, 2014, The Musicology of Record Production, Cambridge, Cambridge University Press.

\section{Enregistrements cités}

Allen, Terry, Juarez, Sugar Hill, 2004 [1990].

Antony and the Johnsons, I'm a Bird Now, Beggars Banquet, 2005.

Captain Beefheart, Safe as Milk, RCA, 1969 [2000].

Czukay, Holger, Canaxis, Revisited, 1969 [2006].

Hendrix, Jimi, Band of Gypsys, MCA, 1970 [1997].

Newman, Randy, Good Old Boys, Reprise, 1974 [2002].

Original Dixieland Jass Band (ODJB), « Livery Stable Blues », Frémeaux \& Associés, 1917 [2000].

\section{Liens vers les performances et les paroles des chansons citées}

Newman, Randy, « Rednecks », Rotterdam, 1979, http://www.youtube.com/watch? v=2nGw_vAnqPI. Paroles : http://www.metrolyrics.com/rednecks-lyrics-randy-newman.html. Antony and the Johnsons, « For Today I Am a Boy », Live BBC4, 2006, http://www.youtube.com/ watch?v=hvICM_MU9c8. Paroles : http://www.metrolyrics.com/for-today-i-am-a-boy-lyricsantony-and-the-johnsons.html.

Captain Beefheart, « Electricity », 1968, http://www.youtube.com/watch?v=HE32tcojArI. Paroles : http://www.metrolyrics.com/electricity-lyrics-captain-beefheart.html.

Hendrix, Jimi, « Machine Gun », Live at the Fillmore East, 1969, http://www.dailymotion.com/ video/xopgqz_jimi-hendrix-machine-gun_music\#.Uah-GeCs750. Paroles : http://

www.metrolyrics.com/machine-gun-lyrics-jimi-hendrix.html.

\section{NOTES}

1. Sur la notion de performance, voir également la très bonne synthèse de Julie Mansion-Vaquié : « Noir Désir, ou le corps écorché », dans Barbara Lebrun (2012 : 121-123). Si ce travail porte sur un aspect de la chanson française, il est tout à fait transposable au monde anglophone.

2. L'auteur cite en particulier John Lennon, ce qui n'est pas un mauvais choix, mais il n'explique pas pourquoi. Par ailleurs, s'il est bien un auteur compositeur interprète américain chez qui le texte ne se fond pas dans la musique, c'est plutôt Bob Dylan. Certains critiques vont jusqu'à parler de conflit entre le texte de ses chansons et la musique chez lui. À ce sujet, on consultera avec intérêt Meisel (2009: 153-173 notamment).

3. Voir et écouter: http://www.dailymotion.com/video/xopgqz_jimi-hendrix-machinegun_music.

4. Voir et écouter : http://www.youtube.com/watch?v=2nGw_vAnqPI. 
5. Hirschi parle pour sa part de «canteur ». Pour une analyse très poussée de la persona dans toutes ses dimensions, voir Moore (2012: 179-214). Voir aussi Auslander (2009).

6. Les paroles des chansons et les citations d'articles ou d'ouvrages de langue anglaise sont traduites par l'auteur.

7. Louisiana State University (note de l'auteur).

8. «Parce que, dans les années 1930 , les chanteuses et chanteurs de jazz étaient généralement contraints à chanter des chansons dont les paroles avaient peu de valeur poétique en soi pour la communauté noire, ils trouvèrent d'autres manières de rendre leurs voix médiatisées aussi signifiantes que possible. Les chanteurs noirs trouvèrent dans le scat un moyen de s'échapper, une aire de jeu dans laquelle ils pouvaient laisser libre cours à leur désir d'improvisation et faire tomber les barrières restrictives de paroles banales. "

9. On pourrait ajouter la déclamation à la parole et au chant comme le fait Céline Chabot-Canet (2008 : 146-161) à propos de Léo Ferré. Un exemple américain à rapprocher de Ferré pour la déclamation pourrait être Terry Allen, «La Despedida» (1990: piste $\left.\mathrm{n}^{\circ} 15\right)$. Pour des développements utiles sur voix et chanson, consulter Tagg (2012:343-382) et Deniot et Dutheil (2000).

10. Voir et écouter : https://www.youtube.com/watch?v=hvICM_MU9c8.

11. Sur cette question, voir Richard Middleton (2007 : 116-117 surtout). L'auteur associe Antony au genre de la «torch-song ", d'ancrage très féminin d'ordinaire. Citant Freud sur la question de la névrose obsessionnelle, il signale fort à propos que « l'obsessionnel refuse la différence entre les sexes puisqu'il n'est ni l'un ni l'autre mais les deux à la fois".

12. Pour une approche claire et synthétique de cette question, on peut consulter Lacasse (1999) et Middleton (1990 : 83-93) notamment. Plus récemment, des auteurs anglophones ont envisagé ces questions de manière beaucoup plus extensive : voir notamment Zagorski Thomas (2014 : 20-48 surtout) et Schmidt-Horning (2013 : 78-103, pour l'importance du studio). Sur le rapport entre rythme et technologie, voir Anne Danielsen (dir. 2010). On peut s'étonner en revanche que Tagg n'aborde pas vraiment le sujet dans sa somme musicologique.

13. Voir et écouter : http://www.youtube.com/watch?v=HE32tcojArI.

\section{RÉSUMÉS}

À l'image de ce que la cantologie se propose de faire pour la musique francophone, il nous semble important d'élaborer une méthode d'analyse des chansons populaires américaines qui tienne compte à égalité de la musique, du texte et des voix. La technologie, comprise comme utilisation spécifique des techniques d'enregistrement du studio aura également son importance. En effet, ni la musicologie ni les cultural studies anglo-saxonnes n'ont épuisé la question de la poétique des chansons, à savoir l'étude d'un système fini comparable à un texte à entrées multiples dont aucune ne prévaut sur les autres en termes de statut. L'examen de quelques extraits de chansons servira à illustrer notre propos.

Following the example of what cantology does with French songs, it seems important to us to elaborate a method meant to analyse American popular songs which would take into account the music, the lyrics and voices equally. Technology, defined as a specific use of studio recording techniques, will also matter. Indeed neither musicology nor Anglo-Saxon cultural studies have exhausted the subject of the poetics of songs, meaning the study of a finished system comparable 
to a text with multiple entries in which none of the elements would prevail over the others in terms of status. Some song extracts will be examined in order to illustrate our remarks.

INDEX

Mots-clés : musique populaire américaine, poétique des chansons, cantologie, cultural studies, musicologie

Keywords : american popular music, poetics of songs, cantology, cultural studies, musicology

\section{AUTEUR}

\section{FRÉDÉRIC SYLVANISE}

Université Paris 13, Sorbonne Paris Cité, Pléiade (EA 7338) 